\title{
Specification of the PERFoRM Architecture for the Seamless Production System Reconfiguration
}

\author{
Paulo Leitão*†, José Barbosa*, Arnaldo Pereira* José Barata ${ }^{\ddagger}$ and Armando W. Colombo $§$ \\ * Polytechnic Institute of Bragança, Portugal, Email: \{pleitao, jbarbosa, arnaldo\} @ipb.pt \\ $\dagger$ LIACC - Artificial Intelligence and Computer Science Laboratory, University of Porto, Portugal \\ $\ddagger$ CTS UNINOVA, Faculdade de Ciências e Tecnologia, Universidade Nova de Lisboa, Email: jab@uninova.pt \\ $\S$ University of Applied Sciences Emden/Leer, Germany, Email: awcolombo@technik-emden.de
}

\begin{abstract}
The world is assisting to the fourth industrial revolution, with several domains of science and technology being strongly developed and, specially, being integrated with each other, allowing to build evolvable complex systems. Data digitization, big-data analysis, distributed control, Industrial Internet of Things, Cyber-Physical Systems and self-organization, amongst others, are playing an important role in this journey. This paper considers the best practices from previous successful European projects addressing distributed control systems to develop an innovative architecture that can be industrially deployed. For this purpose, a particular design process has to be addressed in order to consider the requirements and functionalities from various use cases. To investigate the known practices, four use cases are enlighted in this paper, which cover a wide spectrum of the European industrial force, as well as industrial standards to support a smooth migration from traditional systems to the emergent distributed systems.
\end{abstract}

\section{INTRODUCTION}

Globalization allows consumers to have an easy access to producers around the world, becoming irrelevant where producers and consumers are located. Naturally, and alongside with this market globalization, consumers have become more demanding in terms of product customization, quality and cost. In order to face this world-market re-shape, manufacturing companies demand new manufacturing paradigms, techniques and technologies, as well as new business models, for a complete process integration, sustained mainly by a production digitization, massive information exchange and processing [1].

To respond to these industry demands, many national and transnational programs, each one having its own research and innovation strategy, have emerged to support the research on key scientific and technological areas. Industrie 4.0 was established in Germany [2], and later on other European countries followed this vision by promoting similar initiatives, e.g., "Made in Sweden 2030" in Sweden, "La Nouvelle France Industrielle" in France, "Smart Industry" in Netherlands, "Industria Conectada 4.0" in Spain, "Innovate UK" in United Kingdom and "Fabbrica Intelligente" in Italy. Countries from non-european continents are also providing similar roadmaps and funding schemes, particularly the "Industrial Internet" initiative in the United States [3] and the "Made in China 2025" program in China.

These initiatives and roadmaps are based on the adoption of similar technological strategies. Digitization is one of the corner stones in future systems where the goal is the processing of the large amount of data collected from the system assets, namely resources, tools and supply-chain. This is being supported, amongst others, by the use of the Industrial Internet of Things (IoT) to collect massive sensorial data and Big data techniques for the data processing.

Resources, while becoming smarter and pluggable, are able to communicate more effectively with each other, shifting the way these systems are designed, converting the traditional monolithic hierarchical systems into a distributed and horizontal structure, where the diverse components are cooperating and collaborating with each other. Commonly, the aforementioned features are wrapped under a common paradigm, designated as Cyber-Physical Systems (CPS) [4], [5], merging the physical part (i.e. the real world) with the logical part (i.e. the cyber counterpart). Several technological solutions are being advocated as promising to implement CPS solutions and to some extent being already applied. Multi-Agent Systems (MAS) [6], [7] are being used to provide distributed intelligence to the system's components while the Service Oriented Architecture (SoA) principles provide plugability and seamless vertical and horizontal system integration. From a semantic point-of-view, the use of ontologies to define common data structures also supports a seamless integration.

In this context, and aligned with the technological trends and best industrial practices, the PERFoRM project aims to develop an innovative approach to handle the seamless production system reconfiguration, combining the plug-and-produce concept and the human role as a flexibility driver in future production systems. The proposed system also integrates advanced tools to enable the system operationality, namely scheduling, simulation and intelligent decision support. These concepts are aggregated by using a system-wide language that is compliant with legacy systems by using proper adapters. Having this in mind, this paper, based on the best results from previous successful $R \& D$ projects in the field, aims to describe the main pillars of the PERFoRM system architecture.

The rest of the paper is organized as follows: Section II overviews the related work in distributed control systems. Section III describes the requirements established for the design of the distributed system architecture for the seamless production system reconfiguration, which is described in Section IV. Section V details the functionalities of the architectural 
elements, and at the end, Section VI rounds up the paper and points out the future work.

\section{Related WORK in Distributed Control Systems}

Over the past few years, the EU FP7 programme supported several successful projects in the area of agile and plug-andproduce manufacturing, which have contributed to improve the state-of-the-art in the field. In spite of having created a sound conceptual basis, methods and technologies to achieve the true industrialisation, none of these successful projects was individually able to achieve sufficient technical maturity and critical mass to allow large scale industrial uptake of the agile, plug-and-produce system concept. Table I summarizes the evaluation of several relevant projects taking into consideration some criteria regarding the used technologies and features.

SOA principles were embraced by several projects, namely SOCRADES [8], IMC-AESOP [9], ARUM [10], FLEXA [11], SelSus [12], MANUCLOUD [13] and CassaMobile [14], with each one focusing on different ISA95 layers, meaning that they can be applied successfully in industrial automation manufacturing. In a similar way, several projects used MAS as its main technological driver to achieve the decentralization of the control functions, namely GRACE [15], IDEAS [16], PRIME [17] and ARUM projects.

Since several of these projects covered the distributed manufacturing systems, the topics of middleware, interfaces and adapters were also addressed by the majority of them. In particular, PRIME, ReBORN [18] and I-RAMP3 [19] projects have focused on the plugability by considering proper interfaces and adapters. On the other hand, the type of middleware used by the different projects span from Enterprise Service Bus (ESB), e.g., ARUM, to topologies nearest to point-to-point, e.g., FLEXA. In some approaches, e.g., GRACE, IDEAS and PRIME, an agent-based framework was used as middleware to support the interconnectivity among agents, covering only partially the middleware requirements (since they are only able to interconnect agent compliant tools).

The development and integration of high-level tools, e.g., focusing on strategic planning, scheduling and simulation, was touched by ARUM, FLEXA and EMC ${ }^{2}$-Factory [20], some of them developed by using MAS technology and interconnected by using SOA principles, e.g., the agent-based planning developed in ARUM. In another perspective, several projects have considered self-* features, namely GRACE with self-adaptation and self-optimization, IDEAS with selfconfiguration and self-diagnosis, PRIME with self-monitoring, Self-Learning and FRAME with self-learning, ReBORN with self-awareness, SelSus with self-healing and finally, CassaMobile with self-description. At the end, FLEXA, FRAME and PRIME projects addressed the human integration by enabling human interaction.

\section{Collection of Use Cases Requirements}

The validation of the PERFoRM system will be accomplished in 4 uses cases, covering a wide spectrum of the European industrial force, and ranging from home appliances to aerospace and from green mobility to large compressor production. Several requirements were collected from them to be considered in the specification of the system architecture.

The flexibility cluster identifies requirements related to the ability to change production processes in an agile manner, and to adapt the cycle times and their associated costs. The second cluster considers requirements related to reconfigurability, namely the need to have several feedback loops between the different phases of the production process and a decrease of setup times due to the system reconfiguration. More general requirements were also collected, being observable that product and production traceability is mandatory, as well as the automatic gathering of data and the use of simulation tools in the process chain. Furthermore, the integration of systems from different company's departments is of major concern.

\section{System ARChitecture PrinCIPLES}

The design of the system architecture for new innovative production systems should take into consideration the requirements and functionalities defined for the use cases of the PERFoRM project, summarized in Section III. An important assumption is to re-use the results from the previous successful $R \& D$ projects in the field, instead of developing a new architecture from scratch. This assumption also guarantees that the proposed architecture will be backward compatible and aligned with the current state-of-the-art approaches, increasing therefore its industrial adoption possibility.

The analysis of the identified requirements shows that the system architecture should: i) be based on smart and heterogeneous production components, ii) be able to support the seamless system reconfiguration, and iii) be able to enhance planning, simulation and operational features. This section details the design of the system architecture principles taking into consideration these objectives.

\section{A. Network of smart components}

The objective of building a system architecture based on a community of smart and heterogeneous components demands the use of distributed control approaches instead of using the traditional centralized ones. In fact, these approaches are characterized to be rigid and monolithic structures that are not anymore able to face the levels of responsiveness and reconfigurability imposed by the factories of the future. For this purpose, several assumptions are established, each one being concretized using proper methods, approaches and technologies, as illustrated in Table II.

The first assumption supports the idea of how to create a system based on a plethora of distributed and heterogeneous HW devices and SW applications. This can be reached by using service-oriented design principles, encapsulating functionalities as services, which have been proved in SOCRADES, IMC-AESOP and ARUM as a suitable approach for industrial automation. Complementary, this approach follows the IoT paradigm, being necessary to interconnect the production components in a transparent manner, by using standard interfaces (in terms of syntax and semantics) and industrially adopted 


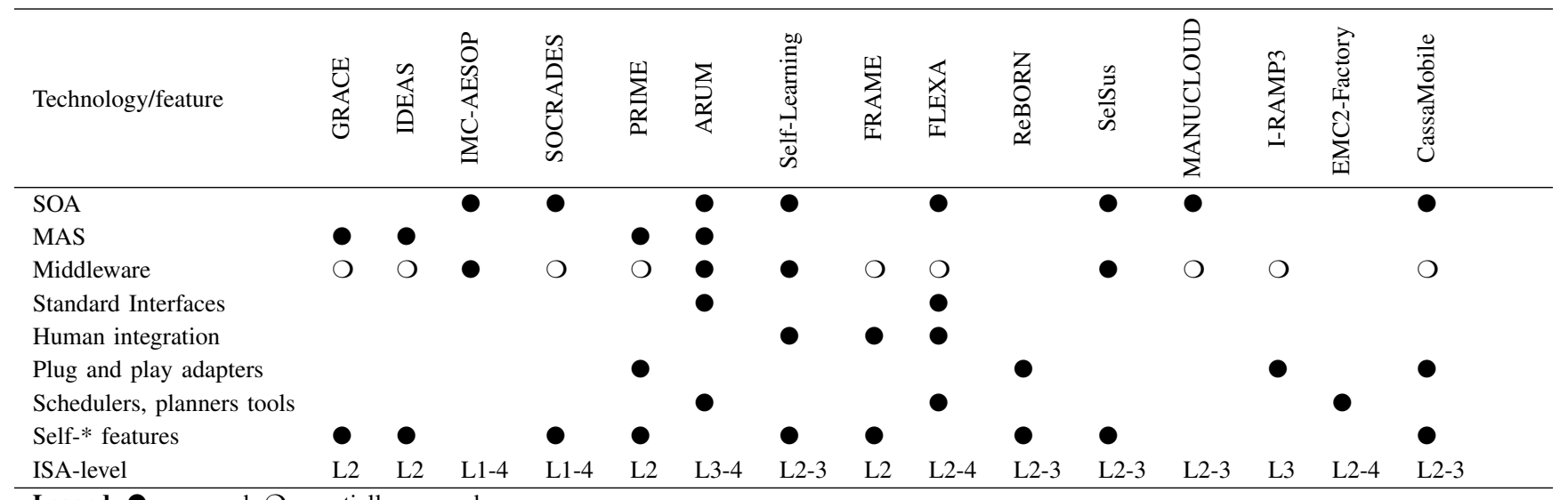

Legend: - - covered; $\bigcirc$ - partially covered

Note: ISA-95 levels are Physical processes (L0), Automation Control (L1), Supervisory Control (L2), Manufacturing Operations Management (L3),

Business Planning and Logistics (L4).

TABLE II. REACHING ASSUMPTIONS TO DEVELOP A SYSTEM BASED ON SMART PRODUCTION COMPONENTS

\begin{tabular}{|c|c|}
\hline Assumptions & How to reach \\
\hline $\begin{array}{l}\text { Distributed and heterogeneous } \\
\text { HW devices and SW applications }\end{array}$ & $\begin{array}{l}\text { Service-oriented design principles } \\
\text { Aggregation and composition of services (or skills) } \\
\text { Holonic design principles }\end{array}$ \\
\hline $\begin{array}{l}\text { Interconnectivity in a easy and } \\
\text { transparent manner }\end{array}$ & $\begin{array}{l}\text { Standard interfaces } \\
\text { Adapters for existing interfaces } \\
\text { Industrially adopted M2M protocols }\end{array}$ \\
\hline $\begin{array}{l}\text { Some production components can } \\
\text { be enriched with intelligence }\end{array}$ & $\begin{array}{l}\text { AI methods and particularly MAS } \\
\text { Advanced data analysis }\end{array}$ \\
\hline Integration of Human in the loop & $\begin{array}{l}\text { HMI and mobile devices } \\
\text { Augmented reality technologies }\end{array}$ \\
\hline
\end{tabular}

M2M protocols, covering the several ISA95 automation levels (see Figure 1).

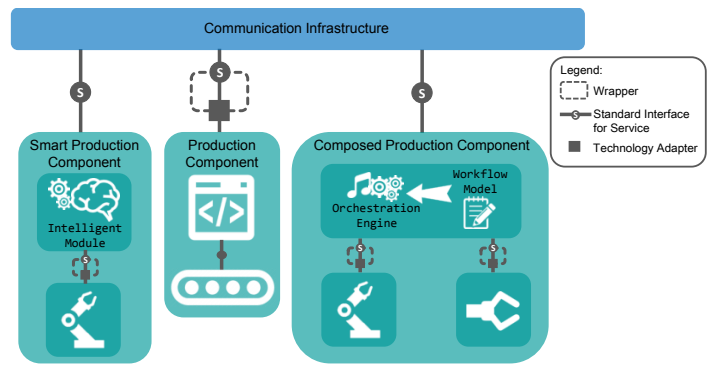

Figure 1: Distributed smart production components.

The creation of system of systems or holistic smart production components can be reached by using the aggregation and composition of atomic services (or skills). In this process, the service orchestration [21] is crucial to sequence and synchronize the execution of the atomic services according to a workflow that represents the business process, providing a high-level interface for such composed process. As an example, illustrated in Figure 1, consider an industrial robot offering the atomic service of "movement" and a gripper offering the atomic service "hold". Composing the two atomic services allows to create a new composed service "pick-andplace". The use of the service composition and orchestration was successfully tested and, thus, approved in the SOCRADES and IDEAS projects.

Some production components can be enriched with intelligence and self-* features to improve their behaviour during run time, e.g., embedding Artificial Intelligence (AI) methods, and particularly MAS technology [6], [7]. In fact, MAS offers an alternative way to design these systems by distributing the automation control functions by several intelligent, autonomous and cooperative agents, providing flexibility, robustness and reconfigurability. Advanced data analytics can be easily integrated in production components, providing a smart layer that allows a fast response to condition changes and the identification of reconfiguration opportunities.

The human integration assumes an important issue as flexibility driver, which is accomplished by interfacing the humans with proper and user friendly Human-Machine Interface (HMI) and mobile devices (e.g., tablets and smartphones) and applying augmented reality technology.

\section{B. Seamless system reconfiguration}

The seamless system reconfiguration is a critical issue in the factories of the future, as identified in Industrie 4.0 initiative. Table III summarizes how this objective can be reached.

\section{TABLE III. REACHING ASSUMPTIONS TO ACHIEVE THE SEAMLESS SYSTEM RECONFIGURATION}

\begin{tabular}{ll}
\hline Assumptions & How to reach \\
\hline \multirow{2}{*}{$\begin{array}{l}\text { Plug and play of production } \\
\text { components on-the-fly }\end{array}$} & $\begin{array}{l}\text { Distributed approaches, e.g., MAS or SOA } \\
\text { Registry and discovery mechanisms } \\
\text { Standard interfaces } \\
\text { Adapters for existing interfaces }\end{array}$ \\
\hline \multirow{3}{*}{ On-the-fly reconfiguration } & $\begin{array}{l}\text { Plug-and-produce concepts } \\
\text { Self-* mechanisms } \\
\text { Reconfiguration boundaries and nervousness control }\end{array}$ \\
\hline
\end{tabular}

The seamless system reconfiguration requires the capability to add, remove or modify production components on-the-fly, 
i.e. without the need to stop, re-program and re-start again the components. This can be reached by using distributed approaches, e.g., MAS or SOA. These approaches provide flexibility and robustness associated to their decentralized and distributed nature, in opposite to the traditional centralized control approaches, which are built up a central node.

Aiming to reach a truly system reconfiguration, the plugand-produce concepts should be considered, taking insights, e.g., from the results achieved in PRIME and I-RAMP-3. For this purpose, the plug and play ability of production components can be simplified by using registry and discovery mechanisms, which are inherent to SOA approaches. In fact, entities that want to offer their functionalities, encapsulated as services, should publish these services in a registry repository that acts as a yellow page functionality. The plug-in of new services in the system is easily discovered by the other entities through the use of a service discovering mechanism. For example, consider a system comprising "Process A" and "Process B" that are interacting with an industrial "Robot". In case of system reconfiguration, through replacing the "Process $\mathrm{A}$ " by "Process C", the intelligence (e.g., an agent) of the "Process B" should de-register its service from the service registry and the intelligence of the "Process $\mathrm{C}$ " should register its service. Automatically, and on-the-fly, the intelligence of the industrial robot discovers the new service and adapts its internal behaviour to start interacting with the new plugged "Process C".

The use of standard interfaces to describe these services in a transparent manner and adapters to convert the existing interfaces to the standard interfaces language, ensure the transparent interconnectivity of these components.

Self-organization mechanisms also play an important role for the system reconfiguration, namely considering the behavioural and structural perspectives, which provides different scopes and time response to evolution [22]. In this field, the reconfiguration boundaries, nervousness and chaos control should be considered.

\section{Enhancing planning, simulation and operational features}

Existing legacy systems focusing planning, simulation and operational features must be integrated and also co-exist with advanced tools taking advantage of powerful computational algorithms and technologies. Table IV summarizes how this objective can be achieved.

TABLE IV. REACHING ASSUMPTIONS TO ENHANCE PLANNING, SIMULATION AND OPERATIONAL FEATURES

\begin{tabular}{ll}
\hline Assumptions & How to reach \\
\hline $\begin{array}{l}\text { Integrate legacy systems, } \\
\text { such as MES, SCADA and } \\
\text { databases }\end{array}$ & $\begin{array}{l}\text { Standard interfaces } \\
\text { M2M and ESB technologies addressing backbone level } \\
\text { Adapters for existing interfaces }\end{array}$ \\
\hline $\begin{array}{l}\text { Integrate advanced planning, } \\
\text { simulation applications }\end{array}$ & $\begin{array}{l}\text { MAS and cloud technologies } \\
\text { Standard interfaces }\end{array}$ \\
\hline $\begin{array}{l}\text { Seamless data representation } \\
\text { and exchange schema }\end{array}$ & $\begin{array}{l}\text { Standards for the representation of industrial data models } \\
\text { Gateways for data transformation (interconnecting back- } \\
\text { bone and machinery levels) }\end{array}$ \\
\hline
\end{tabular}

The integration of legacy systems, such as databases, ERP (Enterprise Resource Planning), MES (Manufacturing Execution Systems) and SCADA (Supervisory Control and Data Acquisition), is simplified by using standard interfaces, and ESB platforms to implement industrial middlewares addressing the higher ISA95 levels. Adapters are commonly used to interconnect these legacy systems by transforming their internal data models into the standard interfaces data model. Advanced planning, scheduling and simulation applications, e.g., developed using the MAS technology, may also be integrated, but in this case without the need to use adapters since they already follow the PERFoRM standard interfaces.

The seamless data representation and exchange schema is reached by considering industrially adopted data models, e.g., IEC 62264 B2MML (Business to Manufacturing Markup Language), which is a XML implementation of the ISA-95, for the backbone environment, and OPC-UA or IEC 62714 AutomationML data models for the machinery environment. The implementation of gateways that interconnect data models from backbone and machinery levels are also required to ensure a proper data transformation.

\section{Architectural Elements}

As result of considering the assumptions established for the initial three objectives described before, the system architecture for the seamless production system reconfiguration is based on a network of HW devices and SW applications, addressing different ISA95 levels, which exposes their functionalities as services following SOA principles, and are interconnected in a transparent manner by using an industrial middleware, as illustrated in Figure 2. This section depicts the core architectural elements allowing the fulfilment of the aforementioned requirements and functionalities.

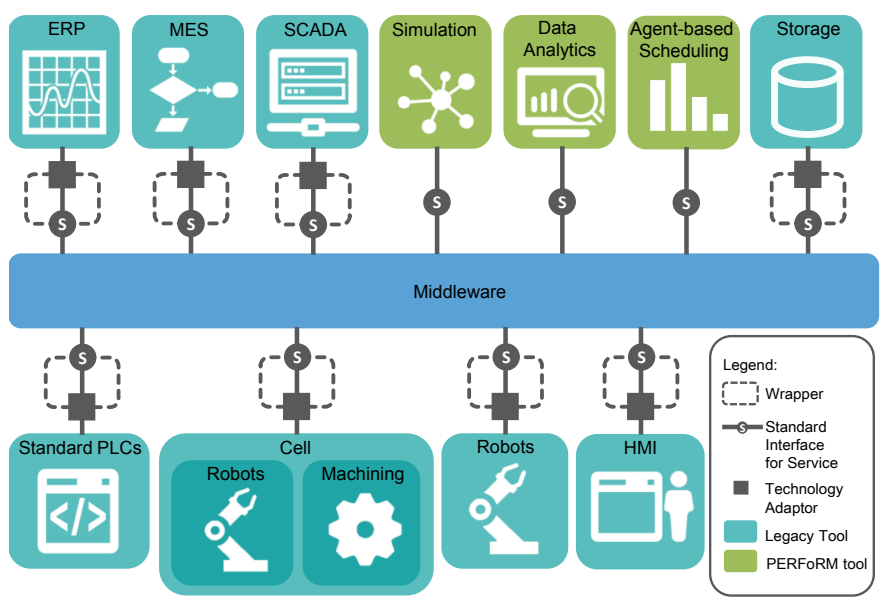

Figure 2: The PERFoRM system architecture.

\section{A. Industrial middleware}

The industrial middleware has a leading role in the system architecture, being responsible to ensure the transparent, secure and reliable interconnection of the diverse HW devices (e.g., robotic cells and Programmable Logic Controllers 
(PLCs)) and SW applications (e.g., MES and SCADA) presented at the PERFoRM ecosystem. Note that some these production components can be enriched with intelligence and others offer composed services based on the aggregation and orchestration of atomic services provided by individual smart production components.

Several requirements can be drawn for the middleware component. Mainly, the middleware should handle the interconnection of these heterogeneous production components by following the service-orientation principles, i.e. exposing their functionalities as services, which will be discovered and requested by the other components. Industrial standards should be considered to simplify the industrial adoption of the proposed solution.

The industrial middleware should offer a set of functionalities that enable the ease of use, tools plugability and performance compliance, namely service registration and discovery, monitor and control the routing of message exchange, prioritization of messages delivery, data transformation, security or exception handling, and data persistence.

The aforementioned list of functionalites ensures a lightweight platform that is easy to be deployable. Some advanced features are envisioned, such as services' orchestration, marshal use of redundant services, services life-cycle monitoring, control deployment and versioning of services and management dashboard (monitoring, audit, logging, metering, admin console, etc.).

\section{B. Standard Interfaces}

Another important architectural element is the use of standard interfaces that allow to interconnect the heterogeneous HW devices and SW applications in a unique, standard and transparent manner, enhancing the seamless interoperability and plugability. The main goal of these interfaces is to develop a common semantic language that every production component is able to interpret and use.

These interfaces should provide a set of functionalities related to a standardized service invocation, i.e. the definition of the list of services to be implemented by the interface, the contract implementation of each service (i.e. the name, input parameters and output parameters), and the definition of the data model handled by the services.

Due to the wide scope of the existing production components, the specification of these standard interfaces must completely integrate different data sources, scopes and with different time scales. Namely, two different worlds should be addressed: the backbone interface (mainly focusing levels L3 and L4 from ISA 95) and the machinery interface (mainly focusing levels L1 and L2 from ISA 95).

\section{Technological adapters}

All manufacturing companies are overfilled with a set of legacy and heterogeneous systems. An innovative architecture is only worthy to be industrially used and really adopted, if the possibility of integrating those legacy systems is presented. In this way, these adapters are responsible to mask the legacy systems by exposing the legacy systems' data/functionalities according to the PERFoRM standard interfaces, i.e. convert the information from the legacy system format to the PERFoRM data model. These adapters involve the HW and/or SW development and are strongly dependent on the selected technology for the middleware.

\section{Human integration}

The integration of the human in the loop is seen as a key factor to improve flexibility. This requirement, and challenge, is considered in the designed architecture by implementing the integration of the human roles through proper HMI. This supports the human integration at different levels, namely at strategic level, e.g. supporting decision-makers to take strategic decisions, and also at operational level, e.g., supporting operators or maintenance engineers to perform their tasks. Since the industrial middleware is independent from the used network technology, locally connected equipment (e.g., typical PCs) but also wireless devices (e.g., tablets or smart phones) may be used to show the gathered and processed data to the user, allowing the interaction to the system.

\section{E. Advanced tools}

Tools particularly designed with advanced algorithms and technologies to support the production planning and scheduling may improve the system performance and reconfigurability. These tools should be PERFoRM compliant, i.e. following the service orientation and using the PERFoRM native interfaces. Examples are the agent-based scheduling, agentbased simulation and intelligent data analytics for real-time visualization, monitoring and processing.

\section{POSITIONING PERFoRM WITHIN INDUSTRIE 4.0}

The digitalization of the shop floor, as proposed by the PERFoRM project, is an approach that completely follows the major characteristics of the Industrie 4.0 platform [23]. The physical (HW / mechatronics) and cyber (SW) views of the shop floor components, which are functionally modularized for guaranteeing, amongst others, re-configurability, are fusioned in one entity. These entities can be recognized as Industrial CPS and they are the cross-scientific and technological subject that communicate and inter-operate using e.g., IoT technology, by exposing and/or consuming "Services" in an operative Internet-of-Services platform.

The smooth, secure and efficient migration from the traditional centralized structures and legacy systems, currently running in industrial environments, to the emergent distributed, agile and plug-and-produce systems, requires a special attention (note that newer devices and/or applications will co-exist with remaining existing systems). This issue may be simplified with the definition of migration methodologies and guidelines, and the adoption of industrial middleware, standard interfaces and repository of wrappers.

The first most important activity in migrating traditional shop floor components into an Industrie 4.0-compliant environment is the specification and transformation of shop floor 


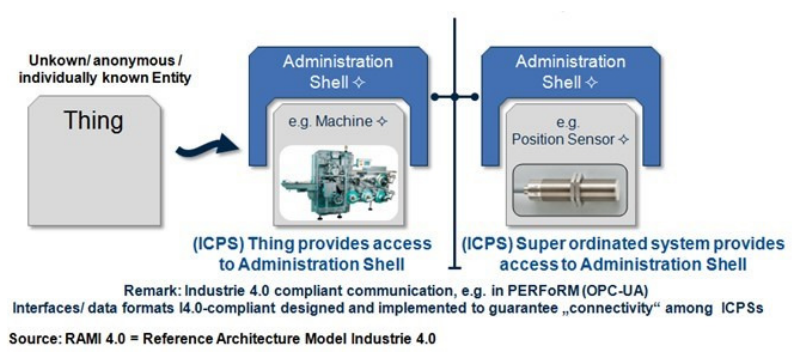

Figure 3: Industrie 4.0 component is an ICPS-component.

components into ICPS-components, i.e., I4.0-components, as briefly described in Figure 3, using the architectural rules addressed by the Reference Architectural Model Industrie 4.0 (RAMI4.0) [24]. In this perspective, robots and machinery became Industrie 4.0 ICPS components comprising the "Thing" itself and the "Administration Shell".

\section{CONCLUSIONS}

This paper has depicted several proposals for distributed control systems where concepts related with CPS and new manufacturing architectural trends were used. From these, the paper has analysed the current best practices and lessons learned, deriving several requirements to be considered in the design of the PERFoRM system architecture. From the end user perspective, several requirements were also considered and accounted, complementing the previous architectural ones.

Merging these two distinct perspectives, the PERFoRM requirements were listed, culminating in architectural principles, such as the use of distributed smart components, seamless system reconfiguration and integrated planning, simulation and operational tools. From a technological point of view, several technologies and approaches were also envisioned, namely the use of service-orientation to expose the system functionalities, the use of a common platform for information exchange, i.e. a middleware, the use of a common language for the specification of standard interfaces, the compliance with legacy systems by means of technology adapters, the use of the human as a flexibility driver and finally the development of advanced planning, simulation and operational tools.

Future work will be devoted to the full specification and implementation of the PERFoRM architecture and its diverse architectural elements.

\section{ACKNOWLEDGEMENT}

This project has received funding from the

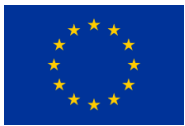

European Unions Horizon 2020 research and innovation programme under grant agreement No 680435 .

\section{REFERENCES}

[1] H. Bauer, C. Baur, G. Camplone, and et. al., "Industry 4.0: How to navigate digitization of the manufacturing sector," tech. rep., McKinsey Digital, 2015.

[2] H. Kagermann, W. Wahlster, and J. Helbig, "Securing the Future of German Manufacturing Industry: Recommendations for Implementing the Strategic Initiative INDUSTRIE 4.0," tech. rep., ACATECH German National Academy of Science and Engineering, 2013.
[3] S.-W. Lin, B. Miller, J. Durand, R. Joshi, P. Didier, and A. C. et al., "Industrial Internet Reference Architecture," tech. rep., Industrial Internet Consortium (IIC), June 2015.

[4] E. A. Lee, "Cyber physical systems: Design challenges," in Proc. of the $11^{\text {th }}$ IEEE International Symposium on Object and Component-Oriented Real-Time Distributed Computing (ISORC'08), p. 363369, 2008.

[5] P. Leitão, A. W. Colombo, and S. Karnouskos, "Industrial automation based on cyber-physical systems technologies: Prototype implementations and challenges," Computers in Industry, Sept. 2015. In Press.

[6] J. Ferber, Multi-Agent Systems, An Introduction to Distributed Artificial Intelligence. Addison-Wesley, 1999.

[7] P. Leitão, "Agent-based distributed manufacturing control: A stateof-the-art survey," Engineering Applications of Artificial Intelligence, vol. 22, pp. 979-991, Oct. 2009.

[8] A. Colombo and S. Karnouskos, "Towards the Factory of the Future: a Service-oriented Cross-layer Infrastructure," ICT Shaping the World: a Scientific View, European Telecommunications Standards Institute (ETSI), pp. 65-81, 2009.

[9] A. W. Colombo, T. Bangemann, and S. Karnouskos, "IMC-AESOP Outcomes: Paving the way to Collaborative Manufacturing Systems,' Proceedings of the 12th IEEE International Conference on Industrial Informatics (INDIN'14), pp. 255-260, 2014.

[10] C. A. Marín, L. Mönch, P. Leitão, P. Vrba, D. Kazanskaia, V. Chepegin, L. Liu, and N. Mehandjiev, "A Conceptual Architecture Based on Intelligent Services for Manufacturing Support Systems," Proceedings of the 2013 IEEE International Conference on Systems, Man, and Cybernetics, SMC, pp. 4749-4754, 2013.

[11] P. Webb and S. Asif, "Advanced Flexible Automation cell," Proceedings of the 6th Innovation for Sustainable Aviation in a Global Environment, pp. 296-301, 2011.

[12] M. S. Sayed, N. Lohse, N. Søndberg-Jeppesen, and A. L. Madsen, "SelSus: Towards a Reference Architecture for Diagnostics and Predictive Maintenance Using Smart Manufacturing Devices," Proceedings of the 13th International Conference on Industrial Informatics (INDIN'15), pp. 1700-1705, 2015.

[13] I. Mezgar and U. Rauschecker, "The Challenge of Networked Enterprises for Cloud Computing Interoperability," Computers in Industry, vol. 65 , no. 4 , p. $657674,2014$.

[14] S. Scheifele, J. Friedrich, A. Lechler, and A. Verl, "Flexible, Selfconfiguring Control System for a Modular Production System," Procedia Technology, pp. 398-405, 2014.

[15] P. Leitão, N. Rodrigues, C. Turrin, and A. Pagani, "Multi-agent system integrating process and quality control in a factory producing laundry washing machines," IEEE Transactions on Industrial Informatics, vol. 11, no. 4, pp. 879-886, 2015.

[16] M. Onori, N. Lohse, J. Barata, and C. Hanisch, "The IDEAS Project: Plug \& Produce at Shop-Floor Level," Assembly Automation, vol. 32 no. 2, pp. 124-134, 2013.

[17] A. Rocha, G. D. Orio, J. Barata, N. Antzoulatos, E. Castro, D. Scrimieri, S. Ratchev, and L. Ribeiro, "An Agent Based Framework to Support Plug and Produce," Proceedings of the 12th IEEE International Conference on Industrial Informatics (INDIN'14), pp. 504-510, 2014.

[18] P. Ferreira, S. Doltsinis, and N. Lohse, "Symbiotic Assembly Systems - A New Paradigm," Procedia CIRP, vol. 17, pp. 26-31, 2014.

[19] G. Gonçalves, J. Reis, R. Pinto, M. Alves, and J. Correia, "A Step Forward on Intelligent Factories: A Smart Sensor-oriented Approach," Proceedings of the IEEE International Conference on Emerging Technology and Factory Automation (ETFA'14), p. 18, 2014.

[20] G. May, B. Stahl, and M. Taisch, "Energy Management in Manufacturing: Toward Eco-factories of the Future A Focus Group Study," Applied Energy, vol. 164, pp. 628-638, 2016.

[21] F. Jammes, H. Smit, J. L. M. Lastra, and I. Delamer, "Orchestration of service-oriented manufacturing processes," in Proceedings of the $10^{\text {th }}$ IEEE International Conference (ETFA'05), pp. 617-624, 2005.

[22] J. Barbosa, P. Leitão, E. Adam, and D. Trentesaux, "Dynamic selforganization in holonic multi-agent manufacturing systems: The ADACOR evolution," Computers in Industry, vol. 66, pp. 99-111, Jan. 2015.

[23] D. Wegener, "Sensors as part of the industrie 4.0," ZVEI - Führungskreis Industrie 4.0, September 2015.

[24] ZVEI, "Industrie 4.0: The Reference Architectural Model Industrie 4.0 (RAMI 4.0)." http://www.zvei.org/Downloads/Automation/ ZVEI-Industrie-40-RAMI-40-English.pdf. 